\title{
Separate "What" and "Where" Decision Mechanisms in Processing a Dichotic Tonal Sequence
}

\author{
Diana Deutsch and Philip L. Roll \\ University of California, San Diego
}

\begin{abstract}
Right-handed subjects were presented with a dichotic tonal sequence, whose basic pattern consisted of three $800-\mathrm{Hz}$ tones followed by two $400-\mathrm{Hz}$ tones on one channel and simultaneously three $400-\mathrm{Hz}$ tones followed by two $800-\mathrm{Hz}$ tones on the other. All tones were $250 \mathrm{msec}$ in duration and separated by 250 -msec pauses. On any given stimulus presentation, most subjects reported the sequence of pitches delivered to one ear and ignored the other. They further tended significantly to report the sequence delivered to the right ear rather than to the left. However, each tone appeared to be localized in the ear receiving the higher frequency, regardless of which ear was followed for pitch and regardless of whether the higher or lower frequency was in fact perceived.
\end{abstract}

When presented with a sensory stimulus, we generate a synthesized percept; for instance, of an object of particular size, shape, color and location, or of a sound of particular pitch, loudness, and duration, emanating from a given point in space. It is generally agreed that the initial stages of this perceptual process involve the abstraction of specific stimulus attributes by highly specialized mechanisms. Indeed, it has even been possible in some cases to demonstrate a gross anatomical separation between pathways relaying information concerning different stimulus attributes. For instance, Schneider (1967) provided strong evidence for an anatomical separation in the visual system between the mechanisms involved in pattern recognition and those involved in object localization. In the case of hearing, Poljak (1926) proposed that the early stages of the auditory pathway involve a ventral route, stbserving localization and orientation functions, and a dorsal rotte, subserving discriminatory functions. Support for this scheme has been provided by recent neuro-

This work was supported by U.S. Public Health Services Grant MH 21001-03.

Requests for reprints should be sent to Diana Deutsch, Department of Psychology, University of California, San Diego, P.O. Box 109, La Jolla, California 92037. physiological findings (Evans, 1974; Evans \& Nelson, 1973a, 1973b).

If we assume, then, that the initial stages of perception involve the analysis of various stimulus attributes by separate mechanisms, the question arises as to how the outputs of these mechanisms are combined to produce an integrated percept. This question becomes particularly intriguing when we consider the case where more than one stimulus is presented at a time. We then have not one value of each attribute to combine together, but several, and we have to know which values to connect with which. The intriguing possibility also emerges that the mechanisms processing information concerning different attributes might arrive at incompatible conclusions, so that stimuli are perceived which have paradoxical properties. The present study demonstrates just such a situation. A sequence of complex tones is presented, each consisting of a pair of pure tones which differ both in pitch and in location. Each simultaneous tone pair gives rise to the fused percept of a single tone. However, the mechanism of fusion for the pitch information differs from that for the localization information, with the result that a perceptual illusion is produced, which is most paradoxical to the listener.

In a previous study, Deutsch (1974a, 1974b) presented listeners with a dichotic se- 
quence of tones which alternated in pitch between $400 \mathrm{~Hz}$ and $800 \mathrm{~Hz}$. Each tone was $250 \mathrm{msec}$ in duration, and there were no gaps between adjacent tones. The identical sequence was presented simultaneously to both ears; however, when the right ear received the $800-\mathrm{Hz}$ tone the left ear received the 400 $\mathrm{Hz}$ tone and vice versa (Figure 1a). Many listeners perceived this sequence as a single tone which alternated in pitch between 400 $\mathrm{Hz}$ and $800 \mathrm{~Hz}$, and which simultaneously alternated in location from one ear to the other (Figure 1b). Further, the apparent locations of the high and low tones remained fixed when the earphones were placed in reverse position. Right-handers tended to hear the high tone on the right and the low

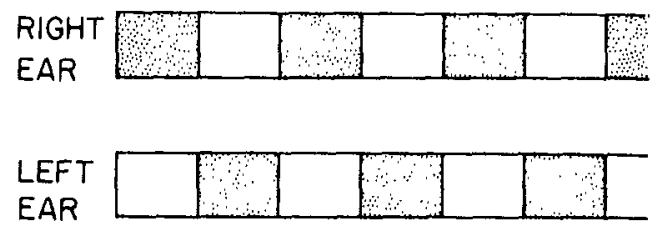

\section{a. STIMULUS}
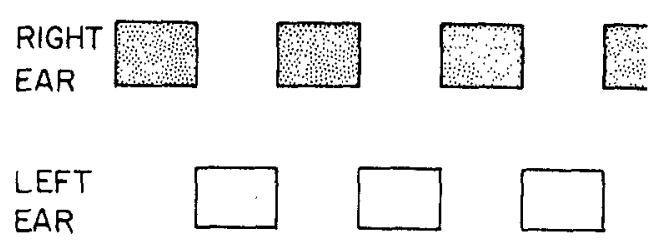

b. PERCEPT

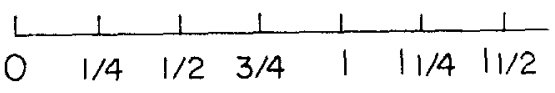

Time in seconds

Figure 1. a. Representation of the dichotic stimulus pattern employed in the experiment by Deutsch (1974a, 1974b). (Filled boxes represent tones of $800 \mathrm{~Hz}$, and unfilled boxes represent tones of 400 $\mathrm{Hz}$. This pattern was repetitively presented without pause for $20 \mathrm{sec}$.) b. Representation of the illusory percept which was most commonly obtained. tone on the left; but left-handers displayed no such tendency. Although the type of percept obtained was found to correlate with handedness, the basis for the illusion was not examined.

The present study was undertaken to test a hypothesized basis for the illusion. It was proposed that this results from the operation of different integrating principles underlying the fused pitch percept and the localization percept. To provide the perceived pitch, one dominant ear exercises a steady suppression on the other, so that only the frequencies arriving at one ear are heard. It was further proposed that in right-handers there is a tendency for the right ear to dominate over the left. However, to provide the perceived localization of each tone, there is no steady dominance of one ear over the other; instead, each tone appears to be localized at the ear receiving the higher frequency. Thus, given a listener who is right-ear dominant, when the $800-\mathrm{Hz}$ tone is delivered to the right ear and the $400-\mathrm{Hz}$ tone to the left, the listener perceives a pitch corresponding to $800 \mathrm{~Hz}$, since this is the tone delivered to the right ear, and the listener also localizes it in the right ear, since this ear is receiving the higher frequency. However, when the 800 $\mathrm{Hz}$ tone is delivered to the left ear and the $400-\mathrm{Hz}$ tone to the right, this listener perceives a pitch corresponding to $400 \mathrm{~Hz}$, since this is the tone delivered to the right ear, but localizes the tone in the left ear, since the left ear is receiving the higher frequency.

This model does not follow from previous findings concerning pitch perception and auditory localization; however, it is not incompatible with them. Concerning the pitch of complex tones, most experiments have involved monaural presentation. Plomp (1964) and Plomp and Mimpen (1968) studied the limits of the ear's frequency resolving power and concluded that listeners were able to distinguish the first five to seven harmonics of a complex tone. Smoorenburg (1970) studied pitch perception of complex tones consisting of two components. He found that subjects were divisible into two categories: They either 
heard the pitches of the individual components or they perceived the complex tone as a whole with a pitch corresponding to the fundamental frequency. In one study involving dichotic presentation, Houtsma and Goldstein (1972) presented subjects with dichotic tonal sequences, in which each dichotic pair consisted of two harmonics of a tone, with a different harmonic presented to each ear. Some subjects responded to this sequence by hearing one partial in each pair, others heard both partials, and yet others retrieved the missing fundamental. A comparison of the response to stimuli delivered to the right and left ears was not reported. Our present model makes the additional assumption that one ear may dominate over the other in determining perceived pitch. This could be mediated by an inhibitory interaction between pathways carrying pitch information from the two ears.

Concerning the localization percept of a single tone produced by the dichotic presentation of two tones, experimental interest has focused on the case where the frequencies presented to the two ears are identical. When these tones are also identical in amplitude, phase, and arrival time, the perceived tone is generally reported as in the center of the head. When the dichotic stimuli differ in amplitude, the perceived tone is displaced towards the ear receiving the higher amplitude signal. Further, when they differ in arrival time, the perceived tone is displaced towards the ear receiving the earlier signal, and differences in phase cause an analogous shift toward the ear leading in phase (Mills, 1972). Theoretical models to explain the apparent location of a single tone as a function of interaural time and intensity differences have also been addressed to the single-frequency case (Jeffress, 1948, 1972; Mills, 1972). For instance, Jeffress hypothesized an array whose individual units were sensitive to specific differences in arrival time between the ears and which were also frequency selective (i.e., they would respond to a given value of interaural delay for only a given frequency value at the two ears). And indeed, neurophysiological evidence for such unițs has been obțained (Goldberg \& Brown,
1969; Moushegian, Rupert, and Langford, 1967). However, in contrast to the singlefrequency case, very little is known about the apparent location of a single perceived tone resulting from the dichotic presentation of two tones of identical amplitude but different frequency. Our present model assumes that, at least for frequency values of $400 \mathrm{~Hz}$ and $800 \mathrm{~Hz}$, the fused percept is lateralized at the ear receiving the higher frequency.

\section{MethoD}

\section{Procedure}

Each subject was individually tested. He was informed that a repetitive tonal sequence would be presented, following which he was to report verbally what he had heard. He was then played the dichotic tonal sequence and gave a verbal report. The position of the earphones was then reversed and the procedure was repeated. The order of earphone placement was strictly counterbalanced across subjects.

\section{Stimulus Parameters}

The basic pattern in the dichotic tonal sequence is shown in Figure 2. It can be seen that this consisted of a sequence of $250-\mathrm{msec}$ tones which were separated by $250-\mathrm{msec}$ pauses. Each tone was either $400 \mathrm{~Hz}$ or $800 \mathrm{~Hz}$; when one ear received $400 \mathrm{~Hz}$ the other ear received $800 \mathrm{~Hz}$ and vice versa. The phase relationship between the tones at the two ears varied randomly. The pattern on Channel A consisted of three presentations of the $800-\mathrm{Hz}$ tone followed by two presentations of the $400-\mathrm{Hz}$ tone. The pattern simultaneously presented on Channel $B$ consisted of three presentations of the $400-\mathrm{Hz}$ tone followed by two presentations of the $800-\mathrm{Hz}$ tone. The entire sequence consisted of the repetitive presentation of this basic pattern 10 times without pause.

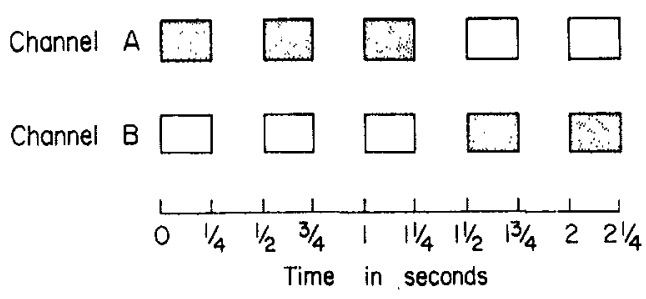

Figure 2. Representation of the basic dichotic stimulus pattern employed in the present experiment. (Filled boxes represent tones of $800 \mathrm{~Hz}$, and unfilled boxes represent tones of $400 \mathrm{~Hz}$. This pattern was repetitively presented 10 times without pause.) 


\section{Apparatus}

Tones were generated as sine waves by two Wavetek oscillators, Model No. 155 (total harmonic distortion less than $1 \%$ between $1 \mathrm{~Hz}$ and $99.9 \mathrm{kHz}$ ), controlled by a PDP 8 computer, and were recorded at equal amplitude on tape. The tape was played to subjects through high-quality earphones at a comfortable listening level ( $75 \mathrm{db}$. SPL).

\section{Subjects}

Forty-four right-handed undergraduates at the University of California, San Diego served as subjects in the experiment and were paid for their services. All had normal hearing in both ears.

\section{Results}

All subjects except three reported sequences consisting of one tone at a time. The percepts of these three subjects are described at the end of this section. For the remaining subjects, the reported localization and pitch patterns were analyzed separately for each earphone position, that is, with Channel $A$ to the left ear and Channel $B$ to the right, and with Channel $B$ to the left ear and Channel $A$ to the right.

\section{Localization Patterns}

For all subjects, each tone appeared to be localized in the ear which received the higher frequency (i.e., the $800-\mathrm{Hz}$ tone). Thus with Channel $A$ to the left ear and Channel $B$ to the right, all subjects reported a repetitive pattern consisting of three tones in the left ear followed by two tones in the right. With Channel B to the left ear and Channel $A$ to the right, all subjects reported a repetitive pattern consisting of two tones in the left ear followed by three tones in the right.

\section{Pitch Patterns}

Thirty-five subjects reported sequences consisting of high and low tones. On any given stimulus presentation, each of these subjects reported a repetitive sequence consisting of either three high tones followed by two low tones or two high tones followed by three low tones. Thus on each occasion the sequences of pitches delivered to one ear was followed and the other suppressed. Table 1 shows the number of sub-
TABLE 1

Numbers of Subjects Following the Sequence of Pitches Presented on Each Channel as a Function of Earphone Position

\begin{tabular}{ccc}
\hline Channel iollowed & $\begin{array}{c}\text { Earphone position } \\
\text { Channel A } \\
\text { to left- } \\
\text { to righnt }\end{array}$ & $\begin{array}{c}\text { Channel B } \\
\text { to left- } \\
\text { Channel A } \\
\text { to right }\end{array}$ \\
\hline $\begin{array}{c}\text { Channel A } \\
\text { Channel B }\end{array}$ & 9 & 24 \\
\hline
\end{tabular}

jects who reported the pitch pattern fed to the right ear and the number reporting the pattern to the left, with earphones positioned each way. It can be seen that the right ear rather than the left was followed in the majority of instances. This tendency was statistically significant for both earphone positions. (With Channel $\mathrm{A}$ to the left ear and Channel $\mathrm{B}$ to the right, $p<.01$, one-tailed, on a binomial test; with Channel $B$ to the left ear and Channel $A$ to the right, $p<.025$, one-tailed, on a binomial test.) There was no effect of order of presentation and no effect of channel $(p>.05$, on binomial tests, in both instances).

It must be noted, however, that the subjects were quite unaware that they were following the pitch information delivered to one ear rather than the other. As described above, each tone appeared to be localized in the ear which received the higher frequency, regardless of whether the higher or lower frequency was in fact perceived. Thus when a low tone was heard, it appeared to be localized, not in the ear to which it was actually presented, but in the opposite ear. This illusory percept appeared very paradoxical to those subjects who followed the pitch information fed to the same ear with earphones positioned both ways. For instance, when a subject who consistently followed his right ear for pitch was presented with Channel $A$ to his left ear and Channel $\mathrm{B}$ to his right, he heard a repetitive sequence whose basic pattern consisted of three low tones in his left ear, followed by two high tones in his right. When the earphone positions were reversed, he now heard a repetitive sequence consisting of two low 
tones in his left ear, followed by three high tones in his right! The procedure of reversing earphone positions therefore appeared to cause the channel to the left to drop a low tone and the channel to the right to add a high tone!

The remaining six subjects who reported repetitive sequences of single tones observed no pitch differences between these tones. These subjects therefore did not channel the pitch information by ear of input. However, as stated above, all these subjects localized each tone at the ear receiving the $800-\mathrm{Hz}$ signal. Thus with Channel $A$ to the right and Channel $B$ to the left, they heard a repetitive sequence consisting of three tones in the right ear followed by two tones in the left ear. With Channel $B$ to the right ear and Channel $A$ to the left, they heard a repetitive sequence consisting of three tones in the left ear followed by two tones in the right ear.

The three remaining subjects reported the following percept. With Channel $A$ to the left ear and Channel $B$ to the right, they reported two high tones in the right ear accompanied by two low tones in the left, followed simply by three low tones in the left ear. With Channel B to the left ear and Channel $A$ to the right, they reported three high tones in the right ear accompanied by three low tones in the left, followed simply by two low tones in the left ear. Thus, when these subjects heard only one tone, its pitch was from the right ear and located where the high tone was presented; but when the right ear was presented with the high tone and the left ear with the low one, both tones were heard simultaneously and located appropriately.

\section{Discussion}

The results of this experiment are in accordance with the perceptual model proposed in the Introduction. First, for most subjects, the presentation of each dichotic tone pair resulted in the fused percept of a single tone, which appeared to be localized in the ear receiving the higher frequency. Second, for most subjects, the sequence of pitches delivered to one ear was perceived and the other suppressed, regardless of how the tones appeared to be localized.

Since the simultaneous tones in each dichotic pair were in an octave relationship, one question that arises concerns the effect of harmonics on the illusion. Since the present experiment involved sine-wave stimuli produced by very low distortion oscillators, any harmonics here must be considered negligible. In further on-line experiments we constructed sequences using either sinewave or square-wave stimuli. The illusion was most clear with sine waves; however, with square waves the percept was still obtained of a single high (complex) tone on the right alternating with a single low (complex) tone on the left. Thus, although the effect is clearest in the absence of harmonics, it is not destroyed by harmonics.

A further question concerns the role of stimulus intensity in the illusion. We have varied the loudness of the sequence within a range of 55 to $85 \mathrm{db}$. SPL and find that the illusion is clearly present throughout this range.

Another issue of interest concerns the dependence of the illusion on the frequency relationship between the alternating tones. We have constructed sequences consisting of alternating tones both within the octave and beyond the octave. It appears that the effect is optimal at a frequency ratio of an octave or its vicinity. As the frequency ratio between the alternating tones decreases from the octave, the fusion effect is gradually reduced, and percepts become increasingly more complex. Percepts also become more ambiguous with frequency ratios larger than an octave. However, the illusion does not depend on a strict octave relationship. With the low tone fixed at $400 \mathrm{~Hz}$, one can vary the frequency of the high tone within $10-15 \mathrm{~Hz}$ in either direction without affecting the percept of a single high tone on the right alternating with a single low tone on the left. It is therefore clear that the effect does not depend on the simultaneous tones being in strict harmonic relationship.

A final question concerns the right-ear advantage obtained for the pitch percept. This pattern may be compared with patterns 
of ear advantage found in other dichotic listening studies involving nonverbal materials. When certain complex nonverbal stimuli are dichotically presented, several investigators reported that right-handers tend to process the information delivered to the left ear better than to the right. This has been found in the case of short excerpts of melodies generated by woodwind or stringed instruments (Kimura, 1964) or by humming (King \& Kimura, 1972), sonar signals (Chaney \& Webster, 1966), and environmental sounds (Curry, 1967; Knox \& Kimura, 1970). However, these studies all employed complex stimuli, and it is unclear which acoustic features were responsible for the patterns of ear advantage obtained. Gordon (1970) failed to obtain a left-ear advantage in recognition of dichotically presented melodies when he generated the stimuli on a recorder. However, he did obtain a left-ear advantage in the recognition of dichotically presented chords generated by an electronic organ. In the chords test, two simultaneous tone pairs were presented, one to each ear, so the stimuli were rich in timbral and simultaneous interval information. This suggests that the left-ear advantage found by Kimura in the melodies test may have been due, not to the sequential relationships between the notes of the melodies, but to timbral or other attributes in the recorded excerpts.

This possibility is strengthened by further studies demonstrating a right-ear advantage in processing simple nonverbal sequences. Halperin, Nachshon, and Carmon (1973) presented subjects with dichotic sequences varying in frequency or duration. They found that as the number of frequency or duration transitions increased from zero to two, ear superiority shifted from left to right. Robinson and Solomon (1974) presented subjects with dichotic rhythmic stimuli, produced by pure tones of invariant pitch. They also obtained a right-ear advantage in recognition of these sequences. Further, Papçun, Krashen, Terbeek, Remington, and Harshman (1974) explored the effect of dichotic presentation of Morse code signals. They found that experienced Morse code operators showed a right-ear superiority in perceiving these sequences. Naive subjects also showed a right-ear superiority, provided that the patterns were restricted to seven or fewer elements. With patterns of more than seven elements, ear superiority shifted from right to left. Papçun et al. hypothesized that when the subjects were able to deal with the individual elements and their sequential relationships, processing then took place in the dominant hemisphere. However, when the stimuli became too complex, subjects were forced to adopt a holistic strategy instead, shifting the processing from the dominant hemisphere to the nondominant. In the present experiment, a very simple pitch sequence was employed, consisting of only two elements. The rightear advantage here obtained is therefore in accordance with the recent findings quoted above.

Recently, Efron and Yund (1974) and $Y$ und and Efron (1975) required subjects to judge chords involving tones of $1,500 \mathrm{~Hz}$ and $1,900 \mathrm{~Hz}$, with a different frequency presented to each ear. When these tones were presented at equal amplitude, some subjects reported hearing both components of the dichotic chord. However, others reported a fused sound that was localized in the center of the head. The apparent pitch of this sound was in many cases dominated by the frequency delivered to one ear rather than to the other. Changing the relative amplitude of the tones resulted in an alteration of the apparent location of the sound toward the ear receiving the higher amplitude signal, while the apparent pitch of the sound could continue to be dominated by the frequency delivered to a given ear. In contrast to results with the present paradigm, which involves the continuous presentation of tonal sequences in a lower frequency range and in an octave relationship, the pattern of ear preference in the Efron-Yund paradigm was not found to correlate with handedness. As a further point of contrast, there is no lateralization effect when tones of $1,500 \mathrm{~Hz}$ and $1,900 \mathrm{~Hz}$ are presented simultaneously at equal amplitude, one to each ear. It would thus appear that the two sets 
of phenomena have different underlying bases.

\section{REFERENCES}

Chaney, R. B., \& Webster, J. C. Information in certain multidimensional sounds. Journal of the Acoustical Society of America, 1966, 40, 455-477.

Curry, F. K. W. A comparison of left-handed and right-handed subjects in verbal and nonverbal dichotic listening tasks. Cortex, 1967, 3, 343-352.

Deutsch, D. An auditory illusion. Journal of the Acoustical Socicty of America, 1974, 55, 518-519. (a)

Deutsch, D. An auditory illusion. Nature, 1974, $251,307-309$. (b)

Efron, R., \& Yund, E. W. Dichotic competition of simultaneous tone bursts of different frequency $\rightarrow$ I. Dissociaton of pitch from lateralization and loudness. Neuropsychologia, 1974, 12, 249-256.

Evans, E. F. Neural processes for the detection of acoustic patterns and for sound localization. In F. O. Schmitt \& F. T. Worden (Eds.), The neurosciences, third study program. Cambridge, Mass. : MIT Press, 1974.

Evans, E. F., \& Nelson, P. G. On the relationship between the dorsal and ventral cochlear nucleus. Experimental Brain Research, 1973, 17, 428-442. (a)

Evans, E. F., \& Nelson, P. G. The responses of single neurones in the cochlear nucleus of the cat as a function of their location and the anaesthetic state. Experimental Brain Research, 1973, 17, 402-427. (b)

Goldberg, J. M., \& Brown, P. B. Response of binaural neurons of $d o g$ superior olivary complex to dichotic tonal stimuli: Some physiological mechanisms of sound localization. Journal of Neurophysiology, 1969, 32, 613-636.

Gordon, H. W. Hemispheric asymmetries in the perception of musical chords. Cortex, 1970, 6 , 387-398.

Halperin, Y., Nachshon, I., \& Carmon, A. Shift of ear superiority in dichotic listening to temporally patterned nonverbal stimuli. Journal of the Acoustical Society of America, 1973, 53, 4650.

Houtsma, A. J. M., \& Goldstein, J. L. The central origin of the pitch of complex tones: Evidence from musical interval recognition. Journal of the Acoustical Society of America, 1972, 51, 520-529.
Jeffress, L. A. A place theory of sound localization. Journal of Comparative and Physiological Psychology, 1948, 41, 35-39.

Jeffress, L. A. Binaural signal detection: Vector theory. In J. V. Tobias (Ed.), Foundations of modern anditory theory (Vol. 2). New York: Academic Press, 1972.

Kimura, D. Left-right differences in the perception of melodies. Quarterly Journal of Experimental Psychology, 1964, 16, 355-358.

King, F. L., \& Kimura, D. Left-ear superiority in dichotic perception of vocal nonverbal sounds. Canadian Journal of Psychology, 1972, 26, 111116.

Knox, C., \& Kimura, D. Cerebral processing of nonverbal sounds in boys and girls. Neuropsychologia, 1970, 8, 117-137.

Mills, A. W. Auditory localization. In J. V. Tobias (Ed.), Foundations of modern auditory theory, (Vol. 2). New York: Academic Press, 1972.

Moushegian, G., Rupert, A. L., \& Langford, T. L. Stimulus coding by medial superior olivary neurons. Journal of Neurophysiology, 1967, 30, 1239-1261.

Papçun, G., Krashen, S., Terbeek, D., Remington, R., \& Harshman, R. Is the left hemisphere specialized for speech, language and/or something else? Journal of the Acoustical Society of America, 1974, 55, 319-327.

Plomp, R. The ear as frequency analyzer. Journal of the Acoustical Society of America, 1964, $36,1628-1636$.

Plomp, R., \& Mimpen, A. M. The ear as frequency analyzer II. Journal of the Acoustical Society of America, 1968, 43, 764-767.

Poljak, S. The connections of the acoustic nerve. Journal of Anatomy, 1926, 60, 465-469.

Robinson, G. M., \& Solomon, D. J. Rhythm is processed by the speech hemisphere. Journal of Experinental Psychology, 1974, 102, 508-511.

Schneider, G. E. Contrasting visuomotor functions of tectum and cortex in the golden hamster. Psychologische Forschung, 1967, 31, 52-62.

Smoorenburg, G. F. Pitch of two-tone complexes. In R. Plomp \& G. F. Smoorenburg (Eds.), Frequency analysis and periodicity detection in hearing. Leiden, Netherlands : Sij thoff, 1970.

Yund, E. W., \& Efron, R. Dichotic competition of simultaneous tone bursts of different frequency-II. Suppression and ear dominance functions. Neuropsychologia, 1975, 13, 137-150.

(Received Apri1 9, 1975) 